\title{
Non-Darcy unsteady MHD Hartmann flow in a porous medium with heat transfer
}

\author{
Hazem Ali Attia ${ }^{1}$, Mostafa A. M. Abdeen ${ }^{2, a}$ and Karem Mahmoud Ewis ${ }^{1}$ \\ 1 Department of Engineering Mathematics and Physics, Faculty of Engineering, El-Fayoum University, 63514 El-Fayoum, Egypt \\ 2 Department of Engineering Mathematics and Physics, Faculty of Engineering, Cairo University, 12211 Giza, Egypt
}

Received 17 October 2013, Accepted 26 June 2015

\begin{abstract}
The time varying magneto-hydrodynamic (MHD) Hartmann non-Darcy flow with heat transfer through a porous medium of an electrically conducting, viscous, incompressible fluid between two infinite parallel insulating porous plates is studied. A non-Darcy model that obeys the Forchheimer extension is assumed for the characteristics of the porous medium. A uniform suction and injection as well as an externally applied uniform magnetic field are applied in the direction normal to the plates where a uniform and constant pressure gradient is imposed in the axial direction. The two plates are kept at different but constant temperatures while the Joule and viscous dissipations are considered in the energy equation. The effect of the magnetic field, the Hall current, the porosity of the medium, and the uniform suction and injection on both the velocity and temperature distributions are studied and interesting results are presented for various values of the existing parameters.
\end{abstract}

Key words: Porous medium / non-Darcy model / Forchheimer model / Hartmann flow / magnetohydrodynamic / hall current / numerical solution

\section{Introduction}

The MHD flow between two parallel plates, known as the Hartmann flow, is a classical problem that has many applications in MHD power generators, MHD pumps, accelerators, aerodynamic heating, electrostatic precipitation, polymer technology, petroleum industry, purification of crude oil and fluid droplets and sprays. Hartmann and Lazarus [1] studied the influence of a transverse uniform magnetic field on the flow of a conducting fluid between two infinite parallel, stationary, and insulated plates. Later, a lot of research work concerning the Hartmann flow was done considering different physical effects [2-10]. In most cases the Hall and ion slip terms were ignored in Ohm's law as they have no marked effect for small and moderate values of the magnetic field. However, the current trend of MHD applications is towards a strong magnetic field, so the influence of electromagnetic force is noticeable [5]. Under these conditions, the Hall current and ion slip are important and they have a marked effect on the magnitude and direction of the current density and consequently on the magnetic force term. Tani [7] studied the Hall effect on the steady motion of electrically conducting and viscous fluids in channels. Soudalgekar et al. $[8,9]$ studied the effect of the Hall currents on the steady MHD Couette flow with heat transfer. The tem-

\footnotetext{
a Corresponding author:

mostafa_a_m_abdeen@hotmail.com
}

peratures of the two plates were assumed either to be constant [8] or to vary linearly along the plates in the direction of the flow [9]. Abo-El-Dahab [10] studied the effect of Hall current on the steady Hartmann flow subjected to a uniform suction and injection at the bounding plates. Attia [11] extended the problem of Abo-El-Dahab to the unsteady state with heat transfer and an applied constant pressure gradient.

Fluid flow in porous media is now one of the most important topics due to its wide applications in both science and engineering $[12,13]$. In most of the previous work, the Darcy model was adopted when studying porous flows. The Darcy law is sufficient in studying small rate flows where the Reynolds number is very small. For larger Reynolds numbers the Darcy law is insufficient and a variety of models has been implemented in studying flows in porous media. The Darcy-Forchheimer (DF) model is probably the most popular modification to Darcian flow utilized in simulating inertial effects [14-17]. It has been used extensively in chemical engineering analysis and also in materials processing simulations. Effect of porosity on transient generalized Couette flow with Hall effect and variable properties has been studied by Abdeen et al. $[18,20]$. The unsteady flow in a porous medium between parallel plates in the presence of uniform suction and injection with heat transfer has been investigated in reference [19]. The effect of porosity on the flow of a dusty fluid between parallel plates with heat transfer has been 
discussed in reference [21]. Attia et al. [22] presented the effect of heat transfer between two parallel porous plates for Couette flow under pressure gradient and Hall current.

In the present work, the time varying Hartmann nonDarcy flow with heat transfer through a porous medium of an incompressible, viscous, electrically conducting fluid between two infinite insulating horizontal porous plates is studied with the consideration of the Hall current. The DF model is used for the characteristics of the porous medium. The fluid is acted upon by a constant pressure gradient in the axial direction, a uniform suction and injection as well as a uniform magnetic field are applied perpendicular to the plates. The induced magnetic field is neglected by assuming a very small magnetic Reynolds number $[4,5]$. The two plates are maintained at two different but constant temperatures and the Joule and viscous dissipations are included in the energy equation. A numerical solution for the governing equations of motion and energy is obtained using the method of finite differences. The effect of the magnetic field, the Hall current, the porosity parameters and the suction and injection on both the velocity and temperature distributions are investigated.

\section{Description of the problem}

The two insulating plates are located at the $y= \pm h$ planes and extend from $x=-\infty$ to $\infty$ and $z=-\infty$ to $\infty$ embedded in a DF porous medium where a high Reynolds number is assumed [14-17]. The lower and upper plates are kept at the two constant temperatures $T_{1}$ and $T_{2}$, respectively, where $T_{2}>T_{1}$. The fluid flows between the two plates under the effect of a constant pressure gradient $d P / d x$ in the axial $x$-direction, and a uniform suction from above and injection from below which are applied at $t=0$ with velocity $v_{\mathrm{o}}$ in the positive $y$-direction. The whole system is subjected to a uniform magnetic field $B_{\mathrm{o}}$ in the positive $y$-direction. From the geometry of the problem, it is evident that all quantities are independent of $x$ and $z$-coordinates apart from the pressure $P$. The existence of the Hall term results in a $z$-component of the velocity. Thus, the velocity vector of the fluid is

$$
\vec{v}(y, t)=u(y, t) \vec{i}+v_{o} \vec{j}+w(y, t) \vec{k}
$$

The initial and boundary conditions are: $u=w=0$ at $t \leq 0, u=w=0$ at $y=-h$ for $t>0$ and $u=0$ and $w=0$ at $y=h$ for $t>0$. The temperature $T(y, t)$ at any point in the fluid satisfies both the initial and boundary conditions $T=T_{1}$ at $t \leqslant 0, T=T_{2}$ at $y=+h$, and $T=T_{1}$ at $y=-h$ for $t>0$. The fluid flow is governed by the momentum equation

$$
\rho \frac{D \vec{v}}{D t}=\mu \nabla^{2} \vec{v}-\vec{\nabla} P+\vec{J} \wedge \vec{B}_{\mathrm{o}}+\vec{F}_{D F}
$$

where $\rho$ and $\mu$ are, respectively, the density and the coefficient of viscosity of the fluid. If the Hall term is retained, the current density $\vec{J}$ is given by

$$
\vec{J}=\sigma\left(\vec{v} \wedge \vec{B}_{\mathrm{o}}-\beta\left(\vec{J} \wedge \vec{B}_{\mathrm{o}}\right)\right)
$$

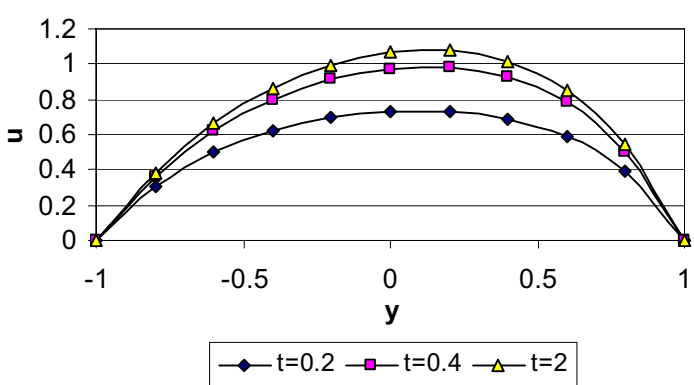

(a)

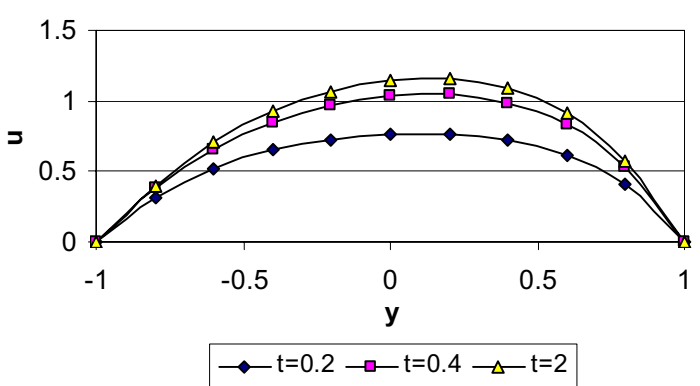

(b)

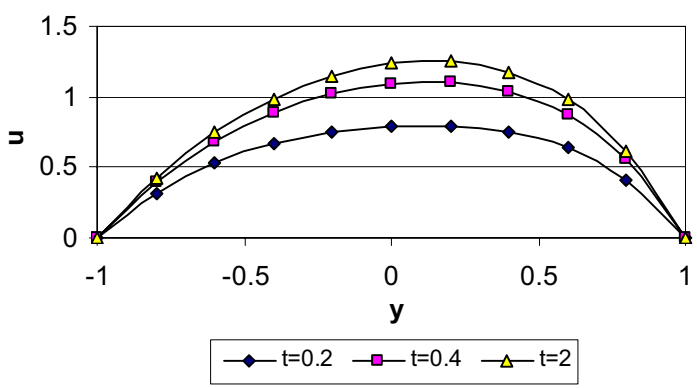

(c)

Fig. 1. Time development of the profile of $u$ at: (a) $m=0$ (b) $m=1$ and (c) $m=3\left(H a=1, M=1, S=1, F_{s}=1\right)$.

where $\sigma$ is the electric conductivity of the fluid, and $\beta$ is the Hall factor [4]. This equation may be solved in $\vec{J}$ yielding

$$
\vec{J} \wedge \vec{B}_{\mathrm{o}}=-\frac{\sigma B_{\mathrm{o}}^{2}}{1+m^{2}}((u+m w) \vec{i}+(w-m u) \vec{k})
$$

where $m=\sigma \beta B_{\mathrm{o}}$, is the Hall parameter [4]. Thus, in terms of Equation (2), the two components of Equation (1) are obtained [12]

$$
\begin{aligned}
\rho \frac{\partial u}{\partial t}+\rho v_{o} \frac{\partial u}{\partial y}= & -\frac{d P}{d x}+\mu \frac{\partial^{2} u}{\partial y^{2}}-\frac{\sigma B_{o}^{2}}{1+m^{2}}(u+m w) \\
& -\frac{\mu}{K_{p}} u-\frac{\rho b}{K_{p}} u^{2} \\
\rho \frac{\partial w}{\partial t}+\rho v_{o} \frac{\partial w}{\partial y}= & \mu \frac{\partial^{2} w}{\partial y^{2}}-\frac{\sigma B_{o}^{2}}{1+m^{2}}(w-m u) \\
& -\frac{\mu}{K_{p}} w-\frac{\rho b}{K_{p}} w^{2}
\end{aligned}
$$

where $K_{p}$ is the permeability of the porous medium and $b$ is the non-Darcian Forchheimer geometrical constant [17]. 

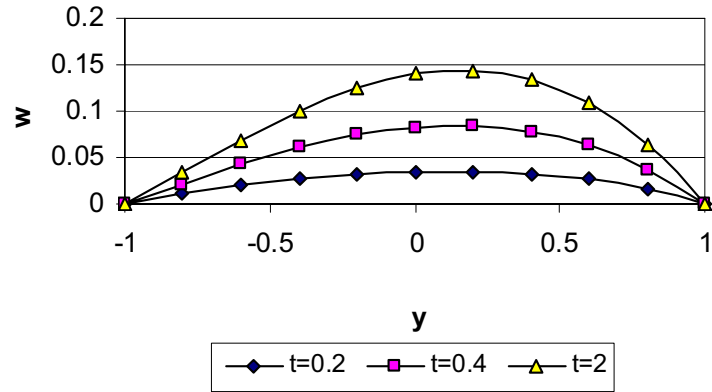

(a)

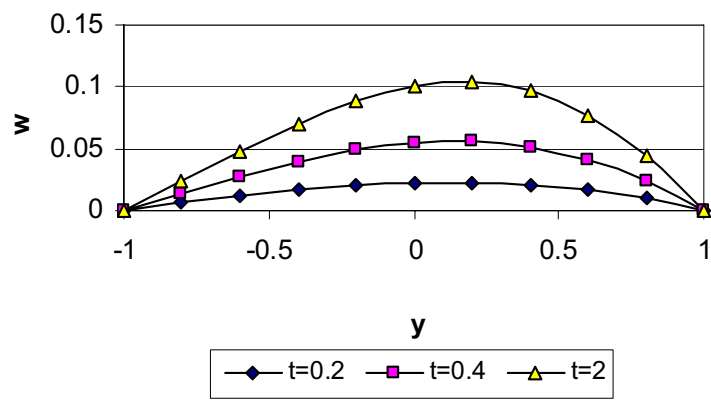

(b)

Fig. 2. Time development of the profile of $w$ at: (a) $m=1$ and (b) $m=3\left(H a=1, M=1, S=1, F_{s}=1\right)$.

The third term in the right side of Equations (3) and (4) both represents the electromagnetic force due to the effect of the Hall current. The last two terms in the right side of Equations (3) and (4) represent the non-Darcy porosity forces.

The temperature distribution is governed by the energy equation [23]

$$
\begin{aligned}
\rho c_{p} \frac{\partial T}{\partial t}+\rho c_{p} v_{o} \frac{\partial T}{\partial y} & =k \frac{\partial^{2} T}{\partial y^{2}}+\mu\left(\left(\frac{\partial u}{\partial y}\right)^{2}+\left(\frac{\partial w}{\partial y}\right)^{2}\right) \\
& +\frac{\sigma B_{o}^{2}}{1+m^{2}}\left(u^{2}+w^{2}\right)
\end{aligned}
$$

where $c_{p}$ and $k$ are, respectively, the specific heat capacity and the thermal conductivity of the fluid. The second and third terms in the right side of Equation (5) represent the viscous and Joule dissipations, respectively. Introducing the following non-dimensional variables

$$
\begin{array}{r}
\hat{x}=\frac{x}{h}, \hat{y}=\frac{y}{h}, \hat{u}=\frac{\rho h u}{\mu}, \hat{w}=\frac{\rho h w}{\mu}, \hat{P}=\frac{\rho h^{2} P}{\mu^{2}}, \\
\hat{t}=\frac{t \mu}{\rho h^{2}}, \hat{T}=\frac{T-T_{1}}{T_{2}-T_{1}}
\end{array}
$$

in addition to the non-dimensional parameters:

$S=\frac{\rho h v_{\mathrm{o}}}{\mu}$, is the suction parameter;

$\operatorname{Pr}=\frac{\mu c_{p}}{k}$, is the Prandtl number;

$E c=\frac{\rho^{2} h^{2}}{\mu^{2} c_{p}\left(T_{2}-T_{1}\right)}$, is the Eckert number;

$H a^{2}=\sigma B_{\mathrm{o}}^{2} h^{2} / \mu$, where $H a$ is the Hartmann number;

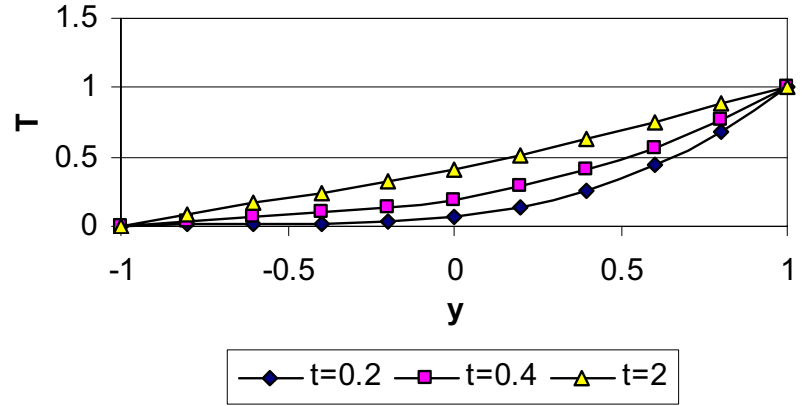

(a)

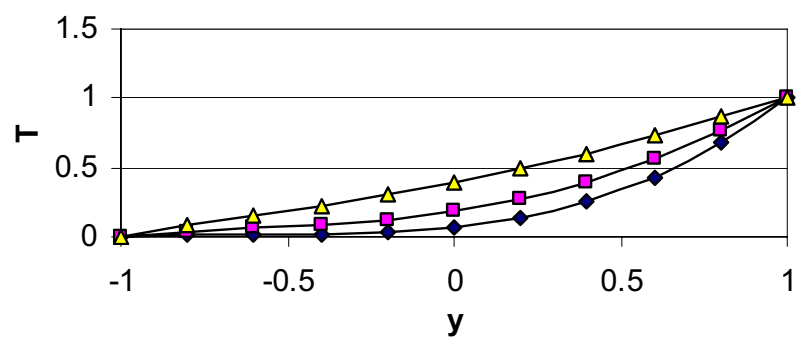

$\neg \mathrm{t}=0.5 \rightarrow-\mathrm{t}=1 \multimap \mathrm{t}=2$

(b)

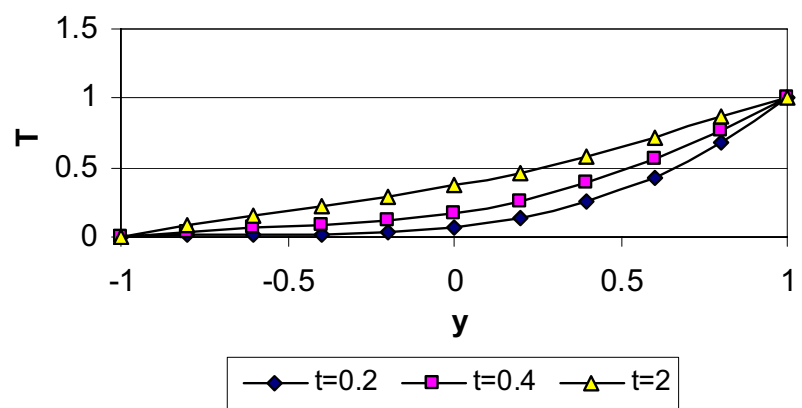

(c)

Fig. 3. Time development of the profile of $T$ at: (a) $m=0$ (b) $m=1$ and (c) $m=3\left(H a=1, M=1, S=1, F_{s}=1\right)$.

$M=h^{2} / K p$ is the porosity parameter;

$F_{s}=h b / K p$ is the dimensionless non-Darcy parameter, the basic Equations (3)-(5) are written as (the hats are dropped for convenience).

$$
\begin{aligned}
& \frac{\partial u}{\partial t}+S \frac{\partial u}{\partial y}=-\frac{\mathrm{d} P}{\mathrm{~d} x}+\frac{\partial^{2} u}{\partial y^{2}}-\frac{H a^{2}}{\left(1+m^{2}\right)}(u+m w) \\
& -M u-F_{s} u^{2} \\
& \frac{\partial w}{\partial t}+S \frac{\partial w}{\partial y}=\frac{\partial^{2} w}{\partial y^{2}}-\frac{H a^{2}}{\left(1+m^{2}\right)}(w-m u)-M w-F_{s} w^{2} \\
& \frac{\partial T}{\partial t}+S \frac{\partial T}{\partial y}=\frac{1}{\operatorname{Pr}} \frac{\partial^{2} T}{\partial y^{2}}+E c\left(\left(\frac{\partial u}{\partial y}\right)^{2}+\left(\frac{\partial w}{\partial y}\right)^{2}\right) \\
& +\frac{E c H a^{2}}{\left(1+m^{2}\right)}\left(u^{2}+w^{2}\right)
\end{aligned}
$$




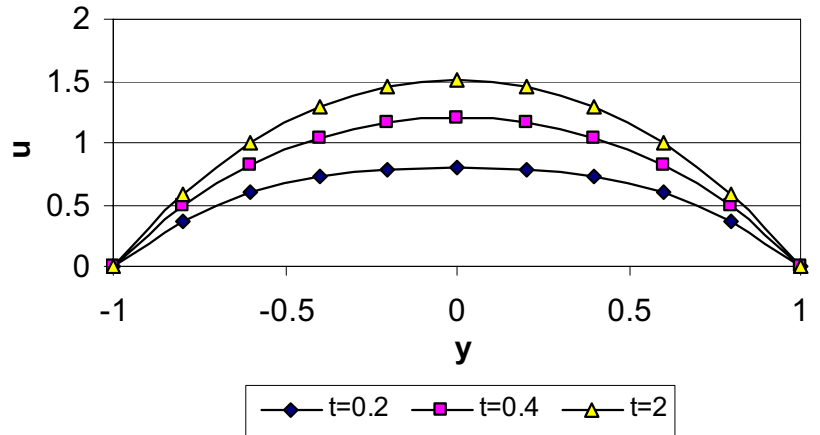

(a)

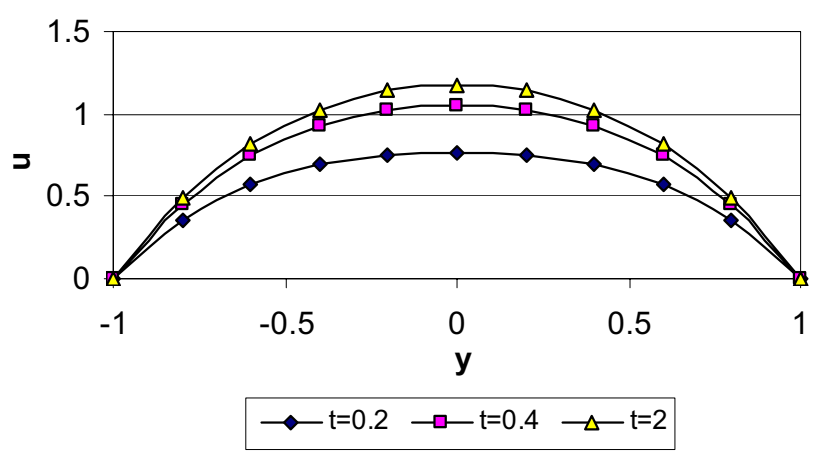

(b)

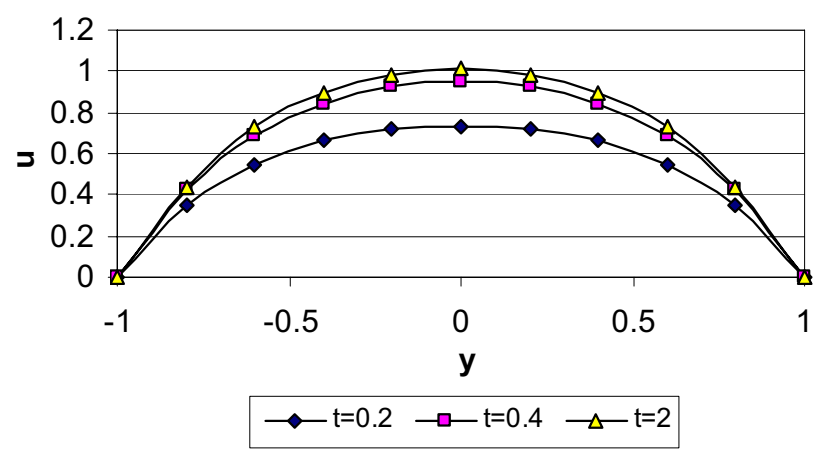

(c)

Fig. 4. Time development of the profile of $u$ at: (a) $F_{s}=0$ (b) $F_{s}=1$ and (c) $F_{s}=2(H a=1, M=1, S=0, m=1)$.

The initial and boundary conditions for the velocity become

$$
\begin{array}{r}
t \leq 0: u=w=0, t>0: u=w=0, y=-1, u=0, \\
w=0, y=1
\end{array}
$$

and the initial and boundary conditions for the temperature are given by

$$
t \leq 0: T=0, t>0: T=1, y=+1, T=0, y=-1
$$

\section{Numerical solution of the governing equations}

Equations (6)-(8) are solved numerically using the finite difference method [24] under the initial and bound-

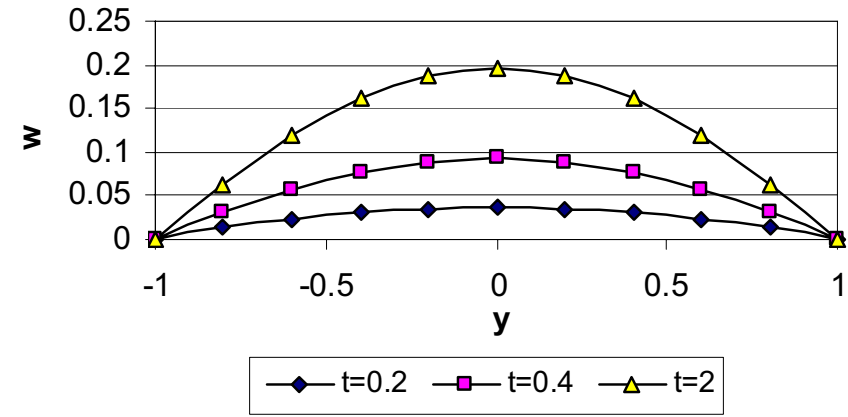

(a)
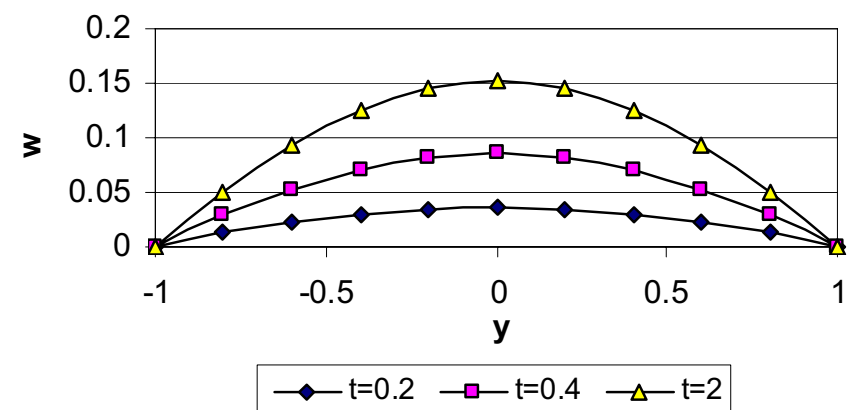

(b)

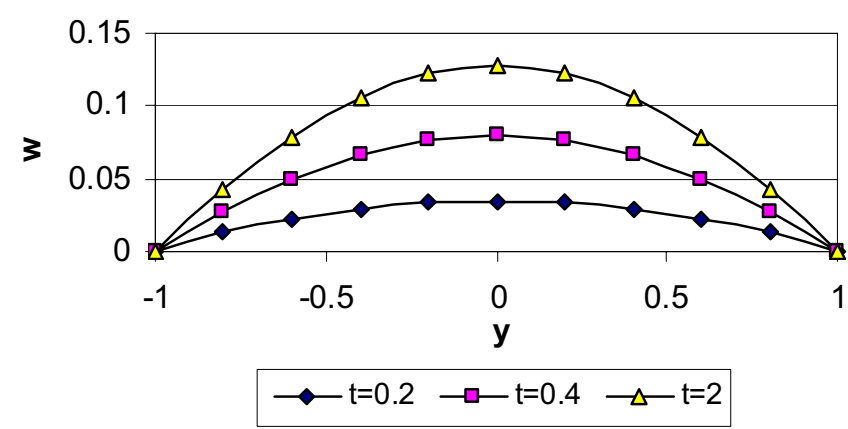

(c)

Fig. 5. Time development of the profile of $w$ at: (a) $F_{s}=0$ (b) $F_{s}=1$ and (c) $F_{s}=2(H a=1, M=1, S=0, m=1)$.

ary conditions (9) and (10) to determine the velocity and temperature distributions for different values of the parameters $H a$ and $S$. The Crank-Nicolson implicit method is applied and the finite difference equations are written at the mid-point of the computational cell and the different terms are replaced by their second-order central difference approximations in the $y$-direction. The diffusion term is replaced with the average of the central differences at two successive time levels. The viscous and Joule dissipation terms are evaluated using the velocity components and their derivatives in the $y$-direction which are obtained from the exact solution. Finally, the block tridiagonal system is solved using Thomas' algorithm. All computations are carried out for $\mathrm{d} P / \mathrm{d} x=5, R e=1$, $\operatorname{Pr}=1$ and $E c=0.2$. 


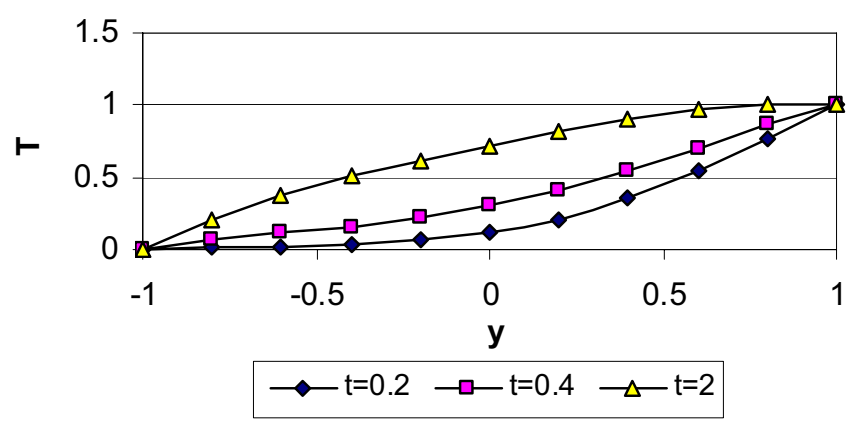

(a)

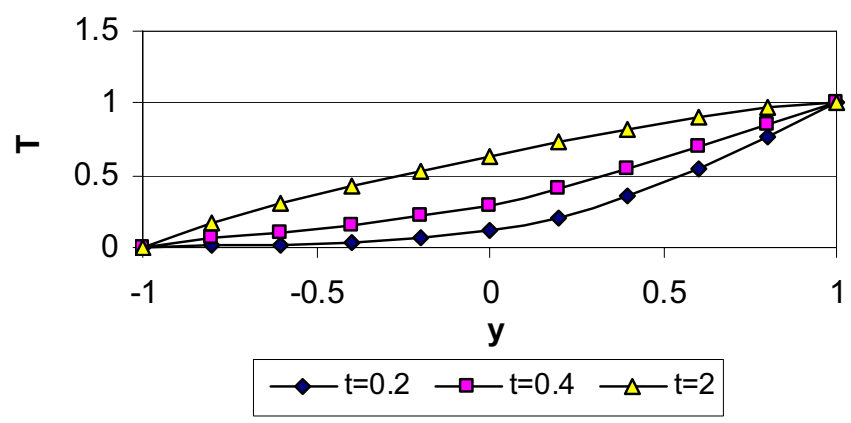

(b)

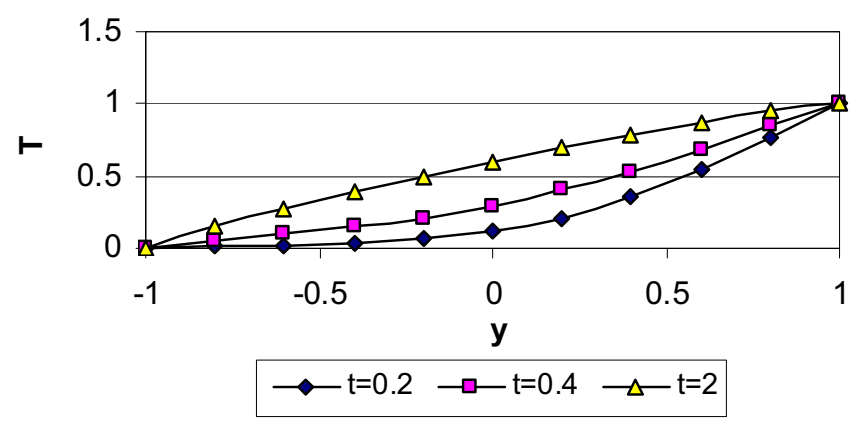

(c)

Fig. 6. Time development of the profile of $T$ at: (a) $F_{s}=0$ (b) $F_{s}=1$ and (c) $F_{s}=2(H a=1, M=1, S=0, m=1)$.

\section{Results and discussion}

Figures 1-3 present the time development of the profiles of the velocity components $u$ and $w$ and the temperature $T$, respectively, for different values of the Hall parameter $m$ and for $H a=1, M=1, F_{s}=1$ and $S=1$. It is seen that the velocity components and temperature reaches the steady state monotonically with time. Also the velocity component $u$ reaches the steady state faster than $w$ which, in turn, reaches the steady state faster than $T$ because $u$ is the source of $w$, while both $u$ and $w$ act as sources for the temperature. It is shown in the Figure 1 that increasing the Hall parameter $m$ increases $u$ due to decreasing the magnetic resistive force. On the other hand, Figure 2 shows that increasing $m$ increases $w$ for small $t$, but decreases it as time develops. The temperature $T$ decreases with increasing $m$ as a

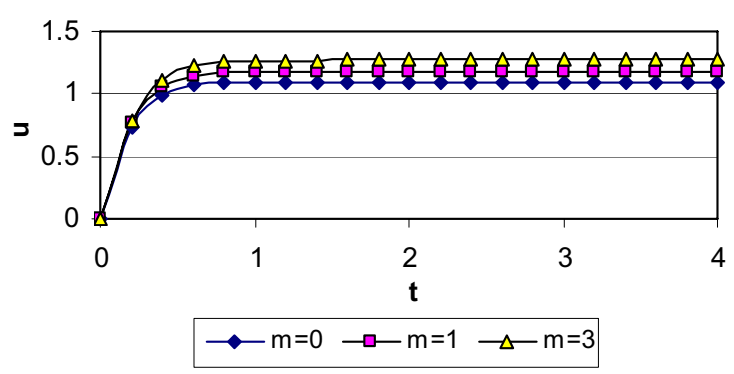

(a)

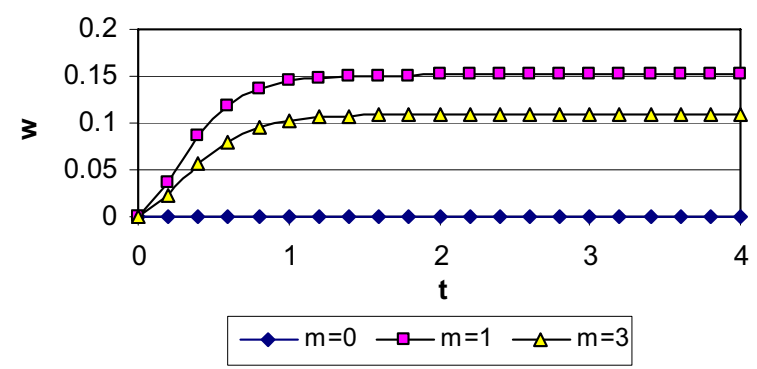

(b)

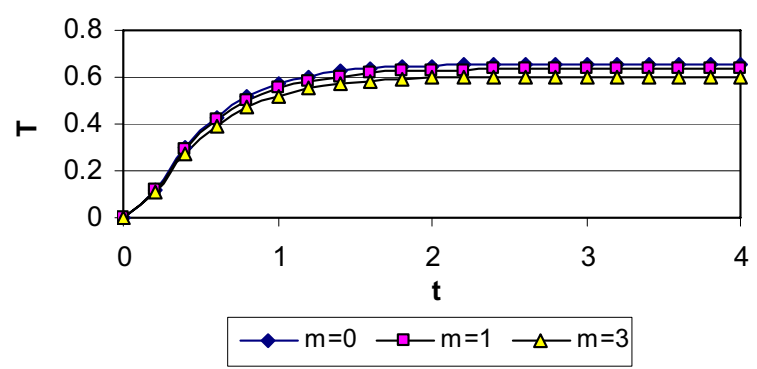

(c)

Fig. 7. Effect of $m$ on the time variation of: (a) $u$ at $y=0$; (b) $w$ at $y=0$; and (c) $T$ at $y=0$. (Ha=1, M=1, S=0, $\left.F_{s}=1\right)$.

result of decreasing the Joule and viscous dissipations as shown in Figure 3.

Figures 4-6 present the time development of the profiles of the velocity components $u$ and $w$ and the temperature $T$, respectively, for different values of the non-Darcy parameter $F_{s}$ and for $H a=1, m=1, M=1$, and $S=1$. It is clear from Figures 4 and 5 that increasing the parameter $F_{s}$ decreases both $u$ and $w$ due to the resistive force of the porous medium. The effect of the non-Darcy parameter $F_{s}$ on $w$ is more pronounced than on $u$. As a result of decreasing both $u$ and $w$, the viscous and Joule dissipations decrease and consequently $T$ decreases.

Figure 7 indicates the time progression of $u$ and $w$ and the temperature $T$ at the centre of the channel $y=0$ for different values of the Hall parameter $m$ and for $H a=1, M=1, F_{s}=1$ and $S=0$. It is clear from Figure $7 \mathrm{a}$ that increasing the parameter $m$ increases $u$ because the effective conductivity $\left(\sigma /\left(1+m^{2}\right)\right)$ decreases with increasing $m$ which reduces the magnetic resistive force on $u$. In Figure 7b, the velocity component $w$ 


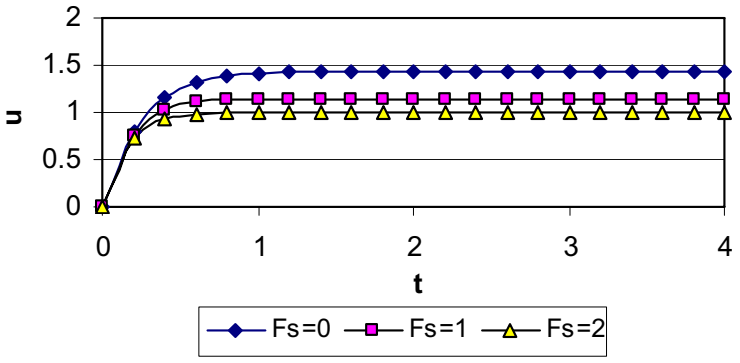

(a)

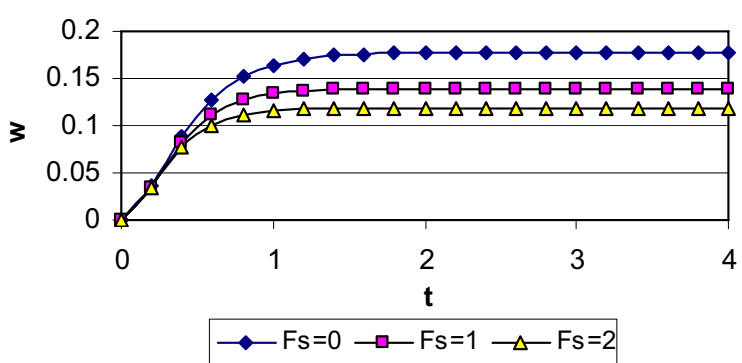

(b)

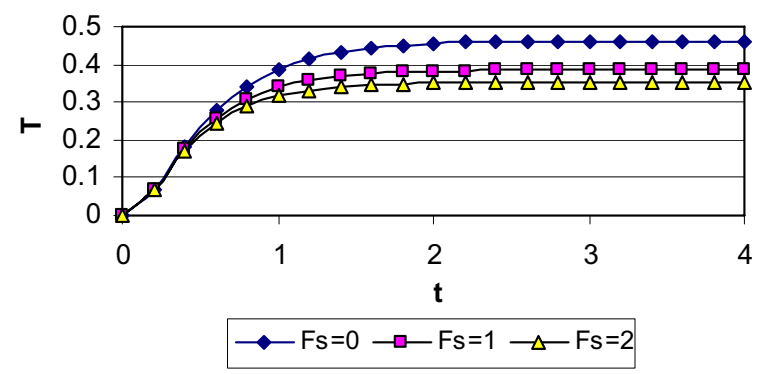

(c)

Fig. 8. Effect of $F_{s}$ on the time variation of: (a) $u$ at $y=0$; (b) $w$ at $y=0$; and (c) $T$ at $y=0 .(H a=1, M=1, S=1$, $m=1)$.

increases with increasing the parameter $m$ slightly $(m=0$ to 1$)$, since increasing $m$ increases the driving force term $\left(m H a^{2} u /\left(1+m^{2}\right)\right)$ in Equation (7) which pumps the flow in the $z$-direction. However, increasing $m$ more decreases the effective conductivity than results in a reduced driving force and then, decreases $w$. It is clear from Figure $7 \mathrm{c}$ that increasing $m$ decreases $T$ for all $t$ due to decreasing the effect of the Joule dissipation.

Figure 8 presents the time progression of $u, w$ and $T$ at the centre of the channel for different values of $F_{s}$ and for $H a=1, m=1, M=1$ and $S=1$. Figures $8 \mathrm{a}$ and $8 \mathrm{~b}$ indicate that increasing $F_{s}$ decreases $u$ and $w$ due to decreasing the damping forces. Figure 8c depicts that increasing $F_{s}$ decreases $T$ due to the decrease in the Joule and viscous dissipations. Figure 9 presents the time progression of $u, w$ and $T$ at the centre of the channel for different values of the suction parameter $S$ and for $H a=1, m=1, M=1$ and $F_{s}=1$. Figures $9 \mathrm{a}$ and $9 \mathrm{~b}$ indicate that increasing the suction decreases both $u$ and $w$ due to the convection of the fluid from regions in the lower half to the centre which has higher fluid speed.

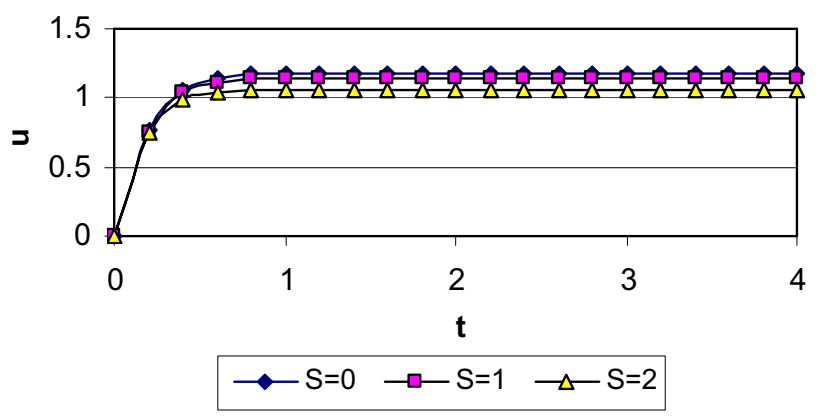

(a)

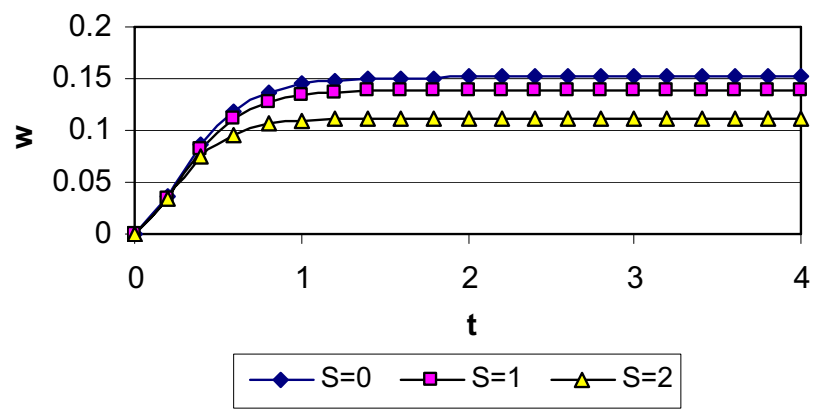

(b)

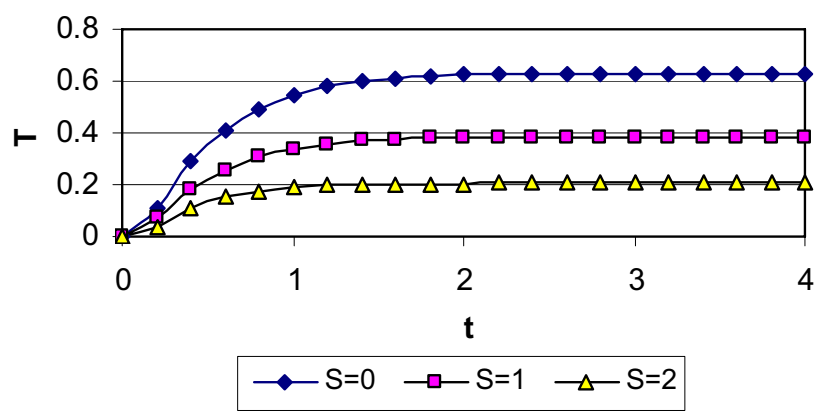

(c)

Fig. 9. Effect of $S$ on the time variation of: (a) $u$ at $y=0$; (b) $w$ at $y=0$; and (c) $T$ at $y=0 .\left(H a=1, M=1, F_{s}=1\right.$, $m=1)$.

Figure 9c shows that increasing $S$ decreases the temperature at the centre of the channel due to the influence of convection in pumping the fluid from the cold lower half towards the centre of the channel.

\section{Conclusion}

The time varying MHD Hartmann flow in a non-Darcy porous medium with heat transfer of an electrically conducting fluid has been studied considering the Hall effect in the presence of a uniform suction and injection. From the present study the following items can be concluded:

- Introducing the Hall term gives rise to a velocity component $w$ in the $z$-direction which affects the main velocity $u$ in the $x$-direction. 
- The effects of the non-Darcy force, the magnetic field, the Hall parameter and the suction and injection velocity on the velocity and temperature distributions are investigated.

- As time develops, increasing the Hall parameter $m$ increases the velocity component $u$.

- By increasing $m$ increases the velocity component $w$ for small $m$ but decreases it for large $m$.

\section{References}

[1] J. Hartmann, F. Lazarus, Kgl. Danske Videnskab. Selskab, Mat.-Fys. Medd. 15 (1937)

[2] I.N. Tao, J. Aerospace Sci. 27 (1960) 334

[3] R.A. Alpher, Int. J. Heat Mass Transfer 3 (1961) 108

[4] G.W. Sutton, A. Sherman, Engineering Magneto hydrodynamics, McGraw-Hill Book Co, 1965

[5] K. Cramer, S.-I. Pai, Magnetofluid dynamics for engineers and applied physicists, McGraw-Hill Book Co, 1973

[6] S.D. Nigam, S.N. Singh, Quart. J. Mech. Appl. Math. 13 (1960) 85

[7] I. Tani, J. Aerospace Sci. 29 (1962) 287

[8] V.M. Soundalgekar, N.V. Vighnesam, H.S. Takhar, IEEE Trans. Plasma Sci. PS 7 (1979) 178

[9] V.M. Soundalgekar, A.G. Uplekar, IEEE Trans. Plasma Sci. PS 14 (1986) 579

[10] E.M.H. Abo-El-Dahab, M.Sc. thesis, Helwan University, Egypt, 1993

[11] H.A. Attia, Can. J. Phys. 76 (1998) 739

[12] A.E. Scheidegger, The physics of flow through porous media, University of Toronto, 1974

[13] M. Kaviany, Principles of heat transfer in porous media, Springer, 1995

[14] S.L. Lee, J.H. Yang, Modelling of Darcy-Forchheimer drag for fluid flow across a bank of circular cylinders, Int. J. Heat Mass Transfer 40 (1997) 3149-3155
[15] J.S. Andrade. Jr., U.M.S. Costa, M.P. Almeida, H.A. Makse, H.E. Stanley, Inertial effects on fluid flow through disordered porous media., Phys. Rev. Lett. 82 (1999) $5249-8252$

[16] N. Jeong, D.H. Choi, C.L. Lin, Prediction of DarcyForchheimer drag for micro-porous structures of complex geometry using the lattice Boltzmann method, J. Micromech. Microeng. 16 (2006) 2240-2250

[17] F. Khani, A. Farmany, M. Ahmadzadeh Raji, F. Addul Aziz Samadi, Analytic solution for heat transfer of a third grade viscoelastic fluid in non-Darcy porous media with thermophysical effects, Commun. Nonlinear Sci. Numer. Simulat. 14 (2009) 3867-3878

[18] M.A.M. Abdeen, H.A. Attia, W. Abbas, W. Abd ElMeged, Effectiveness of porosity on transient generalized Couette flow with Hall effect and variable properties under exponential decaying pressure gradient, Indian J. Phys. 87 (2013) 767-775

[19] H.A. Attia, W. Abd El-Meged, W. Abbas, M.A.M. Abdeen, Unsteady flow in a porous medium between parallel plates in the presence of uniform suction and injection with heat transfer, Int. J. Civil Eng. 12 (2014) 277281

[20] H.A. Attia, W. Abbas, M.A.M. Abdeen, A.E.-D. Abdin, Effect of porosity on the flow and heat transfer between two parallel porous plates with the Hall effect and variable properties under constant pressure gradient, Blug. Chem. Commun. 46 (2014) 535-544

[21] H.A. Attia, W. Abbas, M.A.M. Abdeen, M.S. Emam, Effect of porosity on the flow of a dusty fluid between parallel plates with heat transfer and uniform suction and injection, Eur. J. Environ. Civil Eng. 18 (2014) 241-251

[22] H.A. Attia, W. Abbas, M.A.M. Abdeen, A.A.M. Said, Heat transfer between two parallel porous plates for Couette flow under pressure gradient and Hall current, Sadhana 40 (2015) 183-197

[23] H. Schlichting, Boundary layer theory, McGraw-Hill Book Co, 1968

[24] W.F. Ames, Numerical solutions of partial differential equations, 2nd edn., Academic Press, New York, 1977 